\section{Infection among renal transplant patients in the Kingdom of Bahrain: A ten year retrospective study (2004-2014)}

\section{Abstract}

Objective: Kidney transplantation can be associated with complications encountered during or after operation. Graft survival and immunosuppressant drugs have improved the outcome of transplantation, but infection of grafts have become a leading concern. The diagnosis of infection in such patients is challenging due to the lack of the typical infection indicators like fever since the patients are immunocompromised. This study investigates the extent of infection in transplanted patients, and it will look into the type of microorganisms encountered, their management and outcome.

Methods \& Materials: A retrospective study was carried out between the periods of 1st of January 2004 and 31st of December 2014. All renal transplant patients admitted during this period were investigated according to the reason of admission, and only those with suspected infection were enrolled.

Results: A total of 108 patients included based on suspicion of infection after renal transplant. Some of the patients had recurrent history of infectious over the 10-year period. Out of the 108 patients, 74 received transplant from living donors (68.52\%). The diagnosis on admission was limited to certain signs and symptoms. Urinary tract infections were suspected in $35.19 \%$ of the patients, and pneumonia was diagnosed in $10.19 \%$ of the patients. The majority of patient $(89.96 \%)$ were on maintenance dose of steroids, and were followed by mycophenolate mofetil (MMF), tacrolimus and cyclosporine with a percentage of $81.48 \%, 46.3 \%$ and $25.93 \%$, respectively. Regarding antibiotics, $86.11 \%$ of the admitted patient were started on single antibiotic on admission including those with recurrent admission.
Jameela Al Salman',

Rawan Al Agha², Amani Al Arrayedh²,

D Ahmed Radhi², Maraym Al Alawi², Abeer Khalaf2, Zainab Ebrahim²

\section{Infectious Disease Consultant,} Chairperson of Internal Medicine Department, Salmaniya Medical Complex, Manama/ Bahrain P.O. Box 12

2 Resident in Internal Medicine Department Salmaniya Medical Complex, Manama/ Bahrain P.O. Box 12

Corresponding author:

\section{Jameela Al Salman}

” jalsalman@hotmail.com 
Conclusion: Fever is a common presenting symptom in our population of transplant recipients which reflects the possibility of infection. The majority responded to a single antibiotic tratment.

Key word: Infection transplant patients, Treatment, Bahrain

\section{Introduction}

Kidney transplantation is the process of transplanting the organ to a patient with end stage renal disease and its subdivided according to the donor type. It is indicated in all end stage renal disease patients regardless of the cause.

The first kidney transplant was done in the United States in the 1950 which failed few months later due to lack of immunosuppressant medication. Other attempts followed in France and United Kingdom in 1952 and 1960, respectively [1].

Complications with transplant can be encountered during and after a varying period of operation. It includes graft failure, infection, bleeding and thrombosis. Infections are induced due to the immunosuppressant medication given to the transplant recipient. They are occurring most commonly in the mucocutanous areas (41\%), urinary tract $(17 \%)$ and the respiratory tract (14\%). The most common infectious agents are bacterial (46\%), viral (41\%), fungal (13\%), and protozoan(1\%). The most common viral agents are cytomegalovirus (31.5\%), herpes simplex (23.4\%), and herpes zoster (23.4\%) [2].

Infection of grafts represent a leading concern. It is becoming the main reason for admission through accident and emergency department of transplant patients. Due to the various types of immunosuppressant medications being used a group of pathogens like BK virus and cytomegalovirus which were rarely diagnosed and are being seen more frequently in the post transplant period.

The diagnosis of infection in transplant patients is challenging due to the lack of the typical infection indicators like fever as these patients are immunocompromised. Another challenge is once the source of infection is identified, the physician must alter the immunosuppressant medication to maintain a balance between controlling the causative pathogen and not to lose the graft function. Therefore, antibiotics must be carefully selected as many are nephrotoxic.

In Kingdom of Bahrain, Salmaniya Medical Complex is the only hospital carrying the kidney transplant procedures. A local transplant team was established in the hospital in 1995 and since then there is a continuous work to improve the transplant services. The objective of our study is to study the spectrum of infection observed in the transplanted patients. The study aimed to investigate the pattern of microorganisms encountered, their management and outcome as well as to abstract recommendation to control infection in renal transplant patients..

\section{Patients and Materials}

This 10-year retrospective study was carried out over the periods of $1^{\text {st }}$ of January 2004 and $31^{\text {st }}$ of December 2014. All renal transplant patients admit- 
ted during this period were segregated according to the reason of admission. Only those with impression of infection were enrolled. The end result was of 192 patients who had this impression, however, only the records of 108 patients were found and finally enrolled in the study.

The data of the patients were collected on a written form prepared by the research team. The data included general biographic information, type of infection, type of antibiotics used, and occurrence of any complications, and finally the appearance of rejection and whether it is related to the infection.

\section{Results}

A total of 108 patients were investigated over a 10-year period who suspected to be infected after renal transplant. Some of the patients had recurrent history of suspected infection. The age of patients was ranged between 15 to 75 years old, of these 57 were males and 34 were females.

A total of 74 patients out of 108 patients, received transplant from living donors (68.52\%), six from cadavers (5.56\%), and 27 (25\%) the source of transplant couldn't be verified in their medical records (Figure 1). A total of 26 patients out of 74 received transplant from related donor, 31 from

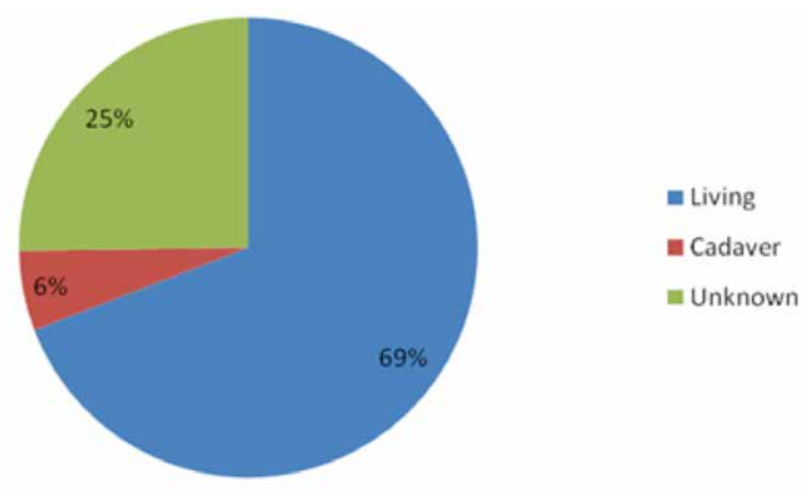

Figure 1. The type of kidney donors. non-related and 17 the source of living donor was unknown in the medical records. All patients received their organs between the periods of 1962 and 2013, but only those who showed signs of infection within the period 2004 and 2014 were enrolled in the study.

The diagnosis of patients on admission was limited to certain differentials with an average period of weeks to few months after the transplant and the first infective episode. A total of 38 /108 (35.19\%) patients were admitted with urinary tract infection (UTI), and 11/108 (10.19\%) whom had history of recurrent UTI on several occasions. These had various significant complains on admission as follow: dysuria $85.71 \%$, fever $65.31 \%$, abdominal or flank pain $24.49 \%$ and $10.20 \%$ with no symptoms but their routine urine culture grew positive organism [Figure 2]. The clinical assessment showed that only 15 / 49 patients had documented fever above or equal to $38{ }^{\circ} \mathrm{C}$, six with abdominal pain and the rest had normal results. The positive results of 38 urine cultures are demonstrated in Figure 2. The majority of urine cultures were sterile in 33 patients (47.83\%; single or multiple admissions), E. coli grew in 11 patients (15.94\%) and ESBL-producing E. coli was detected in 16 patients( 23.19\%) (Figure 2).

Gastroenteritis was diagnosed in $28.70 \%$ of the patients. Stool culture however, was done only for 22 / 31 patients and all were negative for enteric pathogens, except one case of Salmonella spp. Fever was presented in $16.67 \%$ patients, of these $13 / 18$ had fever above or equal to $38^{\circ} \mathrm{C}$.

Pneumonia was diagnosed in 11/108 patients (10.19\%). The majority presented to the emergency with complain of fever, cough and shortness of breath. Three cases were diagnosed as tonsillitis. Chest $x$-ray on admission showed that 7 had lobar infiltrate, three normal $x$-ray and three bilateral infiltrate. The deep tracheal aspiration (DTA)/ sputum was negative for potential pathogens in 8 examined cases. Positive cultures grew the following: one Candida spp., 2 methicilin-resistant S. aureus (MRSA), one Pseudomo- 


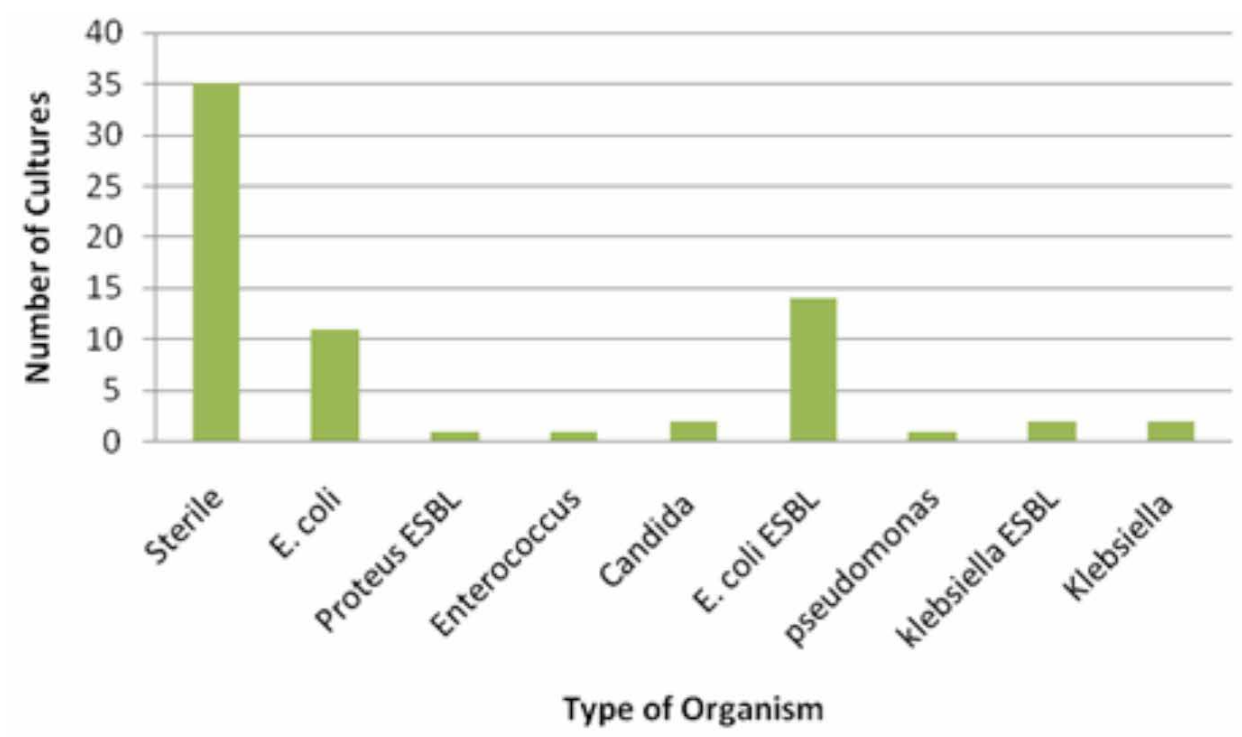

Figure 2. Urine culture results.

nas spp., one multidrug resistance (MDR) Pseudomonas spp., one ESBL-producing Klebsiella spp.

Blood cultures done for 97 patients, and of these, 8 had positive cultures. Three cultures grew E. coli ESBL-producing, and each of the following grew one isolate of MDR Pseudomonas spp., E. coli, MDR Acinetobacter spp., Pseudomonas spp. and Enterobacter spp.

Cytomegalovirus serology test (IgM, IgG) was done for 76 patients. Two out the 76 patients were IgM positive, 16 were positive for IgG, and the rest were negative for both types. Those who were positive for the lgG, the viral load was done for only five of them and it was negative in these patients. All patients were treated with multiple immunosuppressants as shown in Figure $\mathbf{3}$.

Regarding the antibiotics, $86.11 \%$ of the admitted patient were started on single antibiotic on admission including those with recurrent admission, whereas $47.11 \%$ were on multiple antibiotics either as an overlap or at different timing. Ceftriaxone was the main antimicrobial to be prescribed as a single agent (56.5\%) followed by Meropenem (19.4\%) and Pipracillin-Tazobactam (4.6\%). Five patients(24.1\%) were on metronidazole combined with the other antimicrobials. It was prescribed to all patients who had gastrointestinal symptoms and a case of facial cellulitis. Only two patients were treated with antifungal (one with flucanazole and the other with voriconazole). Cotrimaxazole, azithromycin, colistin and ILinezolid were prescribed for different patients in combination with other agents. Six of the patients needed invasive ventilation due to deterioration in their general condition. Total seven patients were shifted to the intensive care unit. Two of them were shifted back to the ward after the condition stabilized and five died. The cause of death according to the death certificate was three septic shock, one cardiogenic shock and one case cardiorenal failure. Another in-patient diagnosed to have viral meningitis in addition to his urinary tract infection and acyclovir was added to his anti-microbial.

None of the patients needed any surgical intervention during their stay except for who underwent ERCP as he was admitted with deranged liver function test, jaundice and urinary tract infection. Fortunately, during the episode of infection none of the patients developed an acute rejection. 


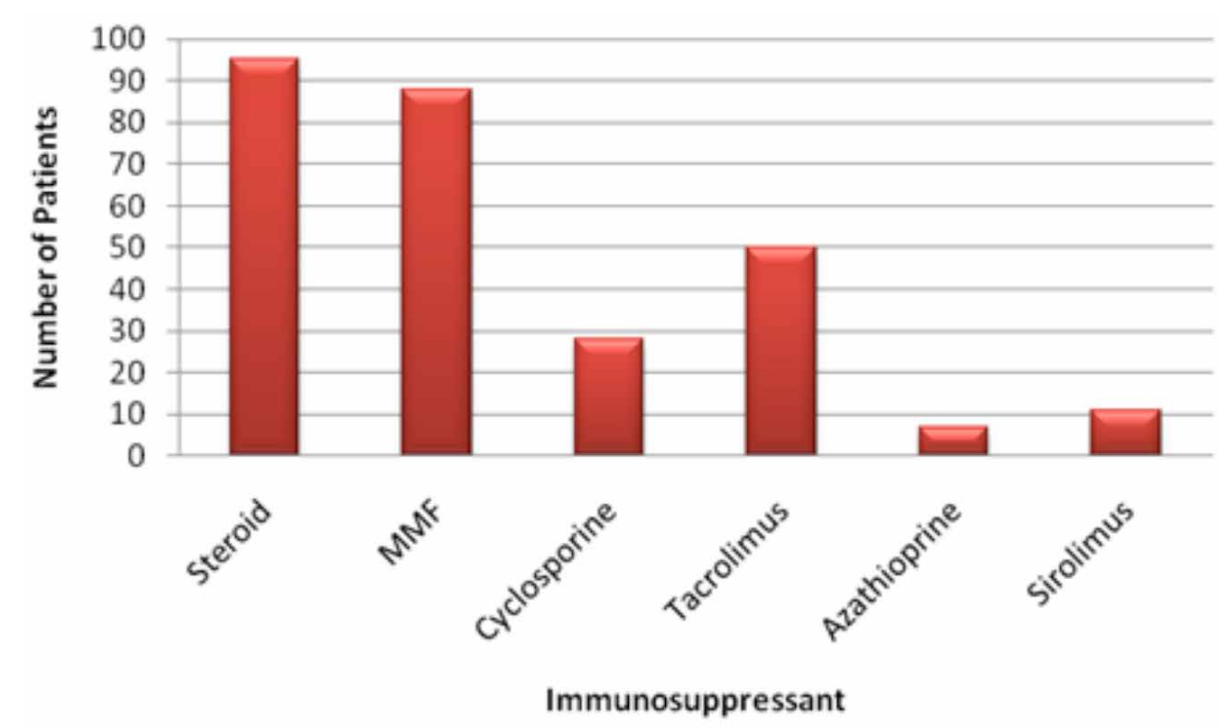

Figure 3. The number of transplant patients on Immunosuppressant.

\section{Discussion}

The renal transplant patients are normally susceptible to infection with a wide range of organisms since they are immunosuppressant [3]. Common exposures to infection in the community are often related to contaminated food and drinks, contact with infected family members or coworkers and others. In addition, they acquire commonly nosocomial infections especially with MDR organisms such as MRSA and ESBL-producing bacteria during their regular hospital visits for clinic follow up.

This study demonstrates that many of our patients have recurrent hospital admissions due to sign or symptom of infection such as fever, urinary or respiratory symptoms. Many studies showed that UTI is the major reason of infection in transplant patients [4]. Our study found that 35\% of the patients had uncomplicated course of UTIs, and they were recovered mostly after treatment with ceftriaxone.

Mitra et al., (2011 ) found that female transplant recipients had significantly more UTIs than males, and the majority of the organisms were Gram-negative bacilli with E.coli is being the most common bacterial type [4]. Since these patients have high incidence of asymptomatic UTIs, it is recommended to give them antibiotic prophylaxis for a period ranging from 6-12 months. The recommended antimicrobial drugs are ciprofloxacin and trimethoprimsulfamethoxazole [5].

Gastroenteritis or diarrhea is also common manifestations among transplant patients. The majority of cases are mild and self limited attack. Prolonged cases can compromise the transplanted kidney by severe dehydration. It is argued that some of the diarrheal episodes are drug related rather than due to infectious agent.

This study demonstrated that the third reason for admission was gastroenteritis associated with diarrhea as main symptom. Upon investigations, all stool cultures were negative for pathogens but the patients were treated for infective diarrhea with a single antibiotic, mostly ceftriaxone and all of the patients showed a good response without any changes in the immunosuppressant medication.

An open nonrandomized, multicenter study done in Belgium during 2006, has reported similar results to our findings about diarrhea, it has identify ap- 
proximately $50 \%$ of patients experienced resolution of severe diarrhea following treatment for infections, or diarrhea associated with medications or due to dietary factors. The study concluded that a large proportion of the severe diarrhea observed in renal transplant recipients is not associated with immunosuppressive therapy and can be treated with antiinfective drugs and other empirical treatments [6].

Fungal infections are opportunistic infections that are rarely cause an infection in immune-competent persons. However, the transplant patients are susceptible candidates for such organisms. Organism like Pneumocystis cranii (PCP) and Aspergillus spp. can result in mortality up to $68 \%$ in immunosupressed transplant recipients [7]. A multinational case-control study in Europe which included 19 institutions and carried out between 2000 and 2013, has reported that 51 kidney transplant recipients were diagnosed to have invasive pulmonary aspergillosis (IPA) after a period of 180 transplant days. Controls recipients were matched by center and date of transplantation, and overall mortality among cases was $60.8 \%$ and $25.0 \%$ and survivors experienced graft loss. Additionally, the study showed that pre-transplant diagnosis of chronic pulmonary obstructive disease (COPD) and delayed graft function were identified as risk factors for IPA. The development of bloodstream infection and acute graft rejection within the three months prior to the diagnosis of IPA was also risk factors during the subsequent period [8]. The present study has not detected any fungal infection among our patients, and this reflects good patient infection control protocols in the hospital and home setting to prevent airborne or person to person transmission of infection plus proper PCP prophylaxis after transplantation.

Renal transplant recipients who are immunosupreseed are susceptible also for viral infections. BK virus is ubiquitous among general population, re- main dormant in healthy persons, and studies had showed positive serum prevalence around $80 \%$ following an asymptomatic primary infection. BK virus becomes active mainly among patients who have received a kidney transplant, in whom the virus can cause tubulo-interstitial nephritis and result in graft failure among approximately 20 to $30 \%$ of nephritic cases [9]. In addition, approximately $1 \%$ to $5 \%$ of transplant patient may develop allogenic nephropathy due to infection with BK virus which can be detected via serum and urine assays. Antivirals are available to treat such viral infection but they can be managed only with immune-suppressant dose reduction [10].

Cytomegalovirus (CMV) is a common cause of mortality and morbidity among transplant population with symptomatic infections are seen in $20 \%$ to $60 \%$. Infection of CMV can be observed directly after discontinuing the prophylactic medication by developing fever, arthritis, hepatitis, meningitis and myalgia or indirect effect through graft rejection, and the virus can be treated successfully by oral and intravenous medications[11].

The present study has not detected any patient with viral infection, except a single patient which has developed viral meningitis and he was treated with acyclovir successfully assuming it had herpes simplex infection.

In conclusion, fever is a common presenting symptom in our examined transplant recipients, and this feature reflects the possibility of microbial infection. The majority responded to a single antibiotic use, and none of them had any of the virulent opportunistic infection. The limitation of our study is the small number of examined transplanted patients. Currently, we recommend to continue applying the same prophylaxis and management protocol for the transplant patients in our center.

There is no conflict of interest. 


\section{References}

1. Hakim, Nadey (2010). Living Related Transplantation. World Scientific. p. 39. ISBN 1-84816-497-1

2. $A B C D$ Renal Transplants, Renal Transplantation Complications from eMedicine (2008). Author: Mert Erogul, MD; Chief Editor: Erik D Schraga, MD. Updated: 5.

3. Fishman JA, Rubin RH: Infection in organ-transplant recipients. N Engl J Med 1998; 338:1741-51.

4. Mitra S, Alangaden GJ. Recurrent urinary tract infections in kidney transplant recipients. Curr Infect Dis Rep 2011; 13:579.

5. Ariza-Heredia EJ, Beam EN, Lesnick TG, Kremers KW, Fernando G. Cosio GF, et al.Urinary tract infections in kidney transplant recipients: role of gender, urologic abnormalities, and antimicrobial prophylaxis. Ann Transplant 2013; 18:195-204

6. B. Maesa K, Hadayab B, de Moorc P, Cambierd P, Peeterse J, Meesterf J, et al. Severe Diarrhea in Renal Transplant Patients: Results of the DIDACT Study. Am J Transplant 2006; 6: 14661472.

7. Schmoldt S, Schuhegger R, Wendler T, Huber I, Söllner $H$, Hogardt $M$, et al. Molecular evidence of nosocomial Pneumocystis jirovecii transmission among 16 patients after kidney transplantation. J Clin Microbiol 2008; 46:966-971.

8. López-Medrano F et.al ; Spanish Network for Research in Infectious Diseases REIPI); Group for the Study of Infection in Transplant Recipients (GESITRA); Spanish Society of Clinical Microbiology and Infectious Diseases (SEIMC); Study Group for Infections in Compromised Hosts (ESGICH); European Society of Clinical Microbiology and Infectious Diseases (ESCMID); Swiss Transplant Cohort Study (STCS). Risk factors associated with early invasive pulmonary aspergillosis in kidney transplant recipients: results from a multinational matched case-control study. Am J Transplant 2016; 12:3311-3582.

9. Lanot A, Bouvier N, Chatelet V, Dina J, Béchade C, Ficheux M, et al. BK virus infections in kidney transplantation. Nephrol Ther. 2016 Apr;12(2):76-85.

10. Hirsch HH, Knowles W, Dickenmann M, Passweg J, Klimkait T, Mihatsch MJ, et al. Prospective study of polyomavirus type BK replication and nephropathy in renal-transplant recipients. N Engl J Med 2002; 347:488-496.

11. Porcu P, Eisenbeis FC, Pelletier PR, Davies AE, Baiocchi AR, Roychowdhuryet $S$, et al. Successful treatment of posttransplantation lymphoproliferative disorder (PTLD) following renal allografting is associated with sustained CD8+ T cell restoration. Blood 2002;100:2341-2348.

\section{Comment on this article:}
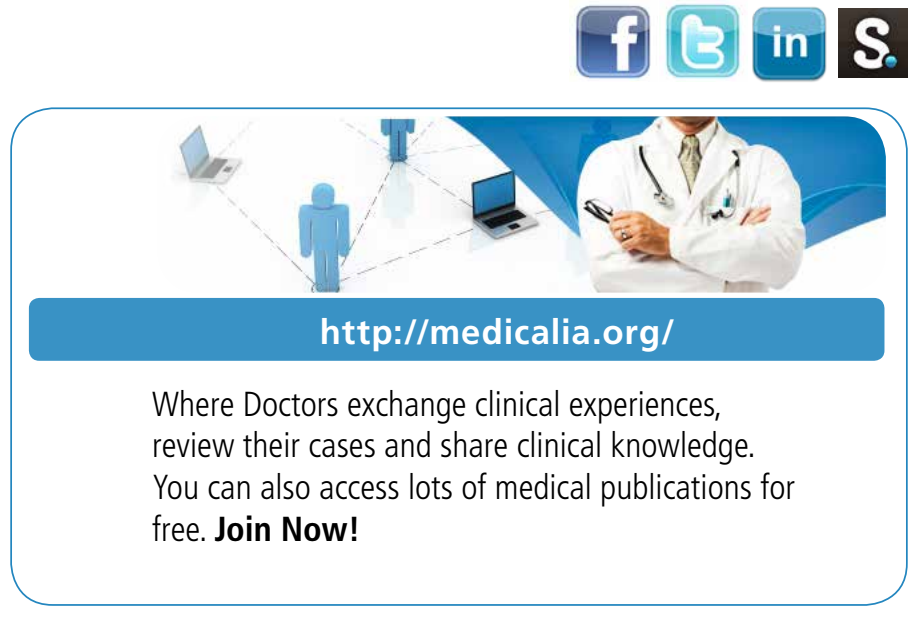

\section{Publish with iMedPub}

http://www.imed.pub

The Journal is an open access peer-reviewed journal that publishes scientific papers about all aspects of antimicrobials. The journal will publish original research articles, reviews, brief reports and case reports dealing with basic and clinical antibacterial agents, antiviral, antiprotozoals, antituberculuous, antifungal and antihelminthes agents.

All manuscripts must be prepared in English, and are subject to a rigorous and fair peer-review process. Accepted papers will immediately appear online.

The journal aims to advance the knowledge, attitude and the research of chemotherapy in the Arabic world in cooperation with international, national scientific and public societies as well as research centers with similar aims and objectives. 